\title{
TIME SERIES CLUSTERING IN LARGE DATA SETS
}

\author{
J. Fejfar, J. Štastný \\ Received: December 17, 2010
}

\begin{abstract}
FEJFAR, J., ŠŤASTNÝ, J.: Time series clustering in large data sets. Acta univ. agric. et silvic. Mendel. Brun., 2011, LIX, No. 2, pp. 75-80

The clustering of time series is a widely researched area. There are many methods for dealing with this task. We are actually using the Self-organizing map (SOM) with the unsupervised learning algorithm for clustering of time series.

After the first experiment (Fejfar, Weinlichová, Štastný, 2009) it seems that the whole concept of the clustering algorithm is correct but that we have to perform time series clustering on much larger dataset to obtain more accurate results and to find the correlation between configured parameters and results more precisely. The second requirement arose in a need for a well-defined evaluation of results. It seems useful to use sound recordings as instances of time series again. There are many recordings to use in digital libraries, many interesting features and patterns can be found in this area. We are searching for recordings with the similar development of information density in this experiment. It can be used for musical form investigation, cover songs detection and many others applications.

The objective of the presented paper is to compare clustering results made with different parameters of feature vectors and the SOM itself. We are describing time series in a simplistic way evaluating standard deviations for separated parts of recordings. The resulting feature vectors are clustered with the SOM in batch training mode with different topologies varying from few neurons to large maps.

There are other algorithms discussed, usable for finding similarities between time series and finally conclusions for further research are presented. We also present an overview of the related actual literature and projects.
\end{abstract}

time series, self-organizing map, clustering

Many objects that we are observing change themselves in time. When we measure properties of these objects we obtain time series - values caught sequentially in time (Wei, Keogh, 2006). This is the reason why time series information retrieving is so important. We are investigating time series in many disciplines including economy, medicine, natural science, engineering, music etc.

Basically, we are investigating two types of tasks on time series: prediction and classification / clustering. There are many problems as need for querying large databases of time series, subjectivity of similarity of time series, data handling problems: sample rates, data formats, missing values. We have plenty of methods for dealing with these problems. In the classification and prediction area we are focusing on the promising concept using artificial neural net- works (ANN). This area is not yet described in all its parts and consequences. Our results in the prediction of economical time series using ANN are presented in the paper (Štencl, Štastný, 2009).

The objective of this paper is our presentation of the huge time series dataset clustering results, with discussing the influence of process properties on those results. It describes the signal-processing phase resulting in a dataset of 1024 time series. It searches for a feedback of time series normalisation on resulting classes. It is also investigating the impact of the Kohonen Self-organizing map properties on resulting classes giving suggestions for setting these variables. Finally it is discussing the complicated fact of the unsupervised SOM performance evaluation. 


\section{METHODS AND RESOURCES}

To meet our goals, we need to perform the classification on large dataset of real data. We are using music recordings as a source of time series. We found Magnatagatune $^{1}$ (Law, 2009) database that can serve as a reference large dataset. It is publicly accessible, so anybody can download this dataset and repeat our experiment. In November 2010 it has more than 25000 clips of recordings. These recordings are split into 16 directories called from " 0 " to " $\mathrm{f}$ ". We use the first 1024 recordings from the first directory called " 0 ". We are presenting unsupervised clustering in this paper. There also exist interesting experiments using semi-supervised time series (electrocardiograms, handwritten documents, manufacturing, and video datasets) classification (Wei, Keogh, 2006).

We are using a simplistic signal processing method dividing the signal into $n$ parts calculating a standard deviation for each part, which results into the $n$-dimensional feature vector for each recording. These feature vectors can be seen as a time series. From the musical point of view they represent information density of a musical piece evolving in the time. This can be used as a musical form descriptor as shows the paper (Fejfar, Weinlichová, Štastný, 2010). A related work searching information dynamics is in the paper (Abdallah, 2007).

Afterwards we perform these feature vectors normalization in the way they vary from -1 to 1 . These normalized feature vectors are clustered with the Self-organizing map (Kohonen, 2001).

The resulting clusters are evaluated with the average Euclidian distance of the observations from the mean of the cluster. At first the average vector is counted for each neuron as a mean of the cluster
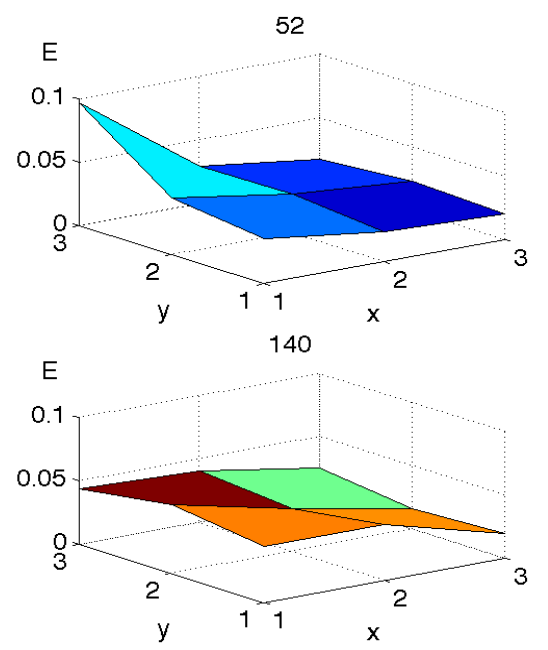

1: Network error, $3 \times 3,3 \mathrm{D}$ feature vector $m_{i}=\frac{\sum_{n=1}^{k} x_{i, n}}{k}$,

where is the dimension of the vector and is the number of observations classified by neuron. After that the average Euclidian distance is counted as

$E=\frac{\sum_{n=1}^{k}|| x_{n}-\mathrm{m}||}{k}$.

\section{RESULTS AND DISCUSSION}

There are three parameters that have to be set in this experiment: the number of dimensions in a feature vector, the number of neurons in the SOM and the number of learning process iterations. We investigate the influence of these parameters on clustering results.

The first decision in our experiment was to set the number of dimensions in a feature vector and the number of neurons in the SOM. We tried 2 different possibilities for each parameter: a small feature vector having 3 dimensions and a larger one with 10 dimensions. For the SOM we experimented with 9 neurons and for the larger one with 100 neurons. Our results can be seen in following figures.

Fig. 1. shows $3 \times 3$ topology of the SOM clustering 3 dimensional feature vectors. The number of iterations is varying from 52 to 1000 . It is interesting that the evolution of the error level is not continuous but rather it is changing significantly around some values of iterations. From 1 to 51 iterations algorithm performs an initial phase, few neurons represent al-
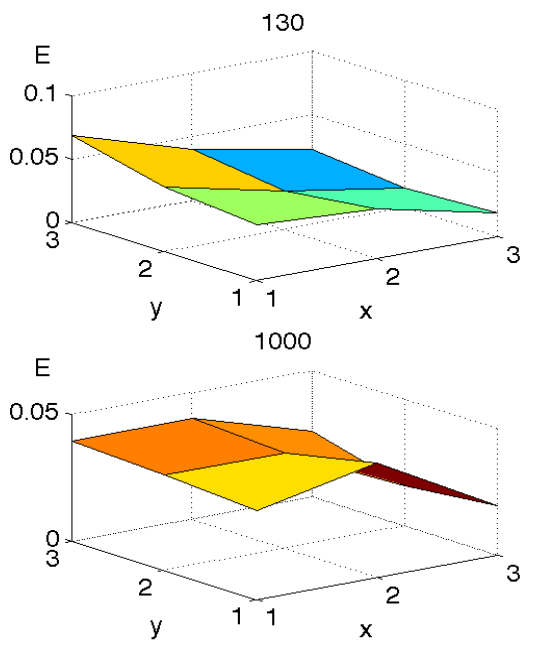

1 http://tagatune.org/Magnatagatune.html 

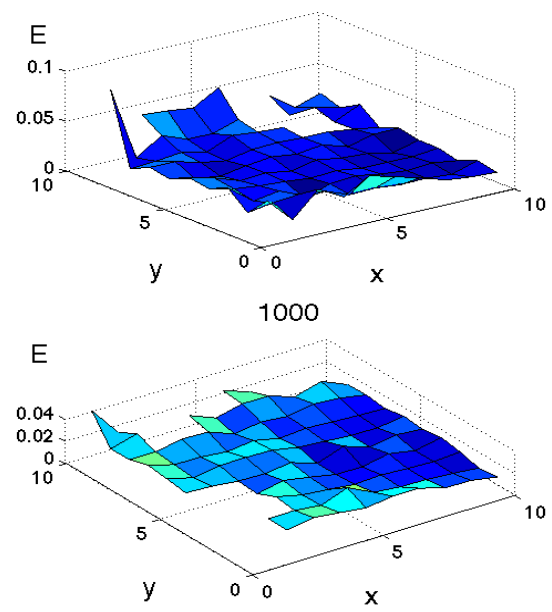

2: Network error, $10 \times 10,3 D$ feature vector
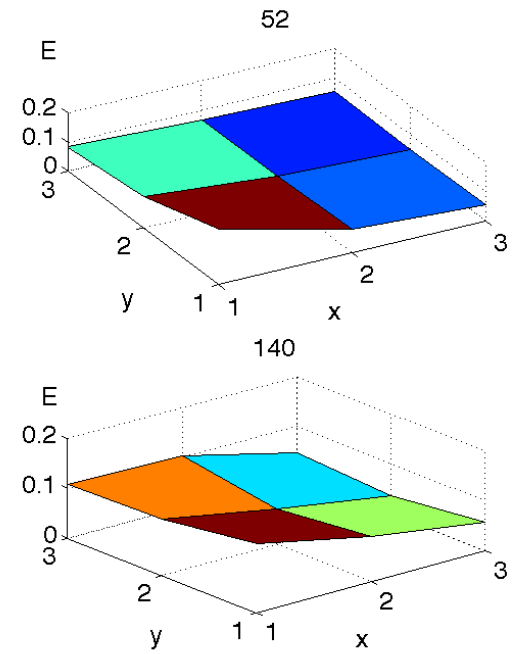

3: Network error, $3 \times 3,10 D$ feature vector

50
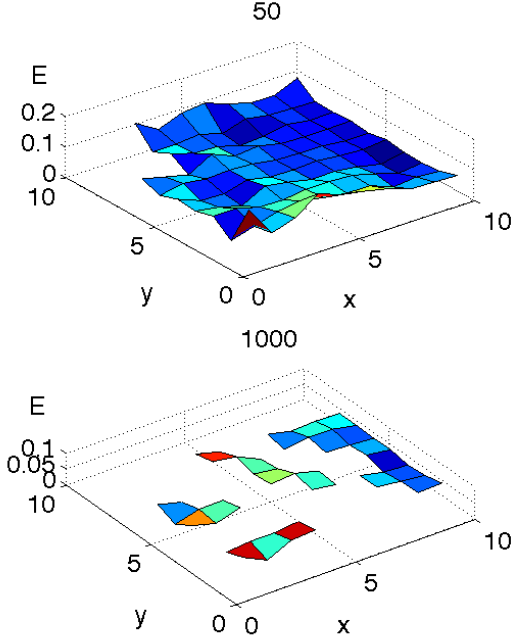

50
200
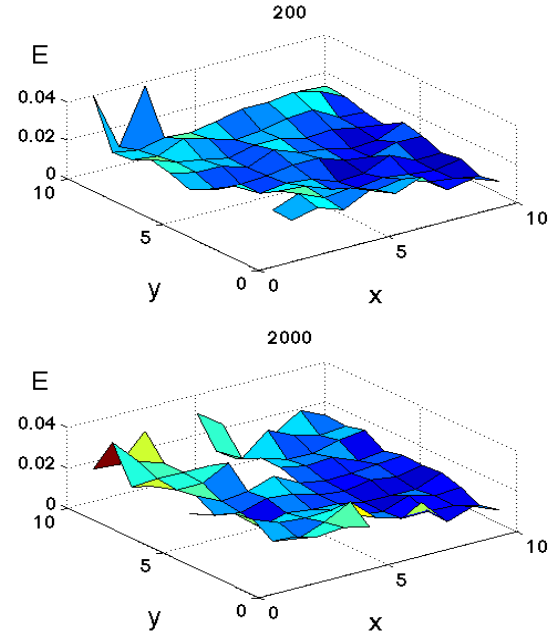

130
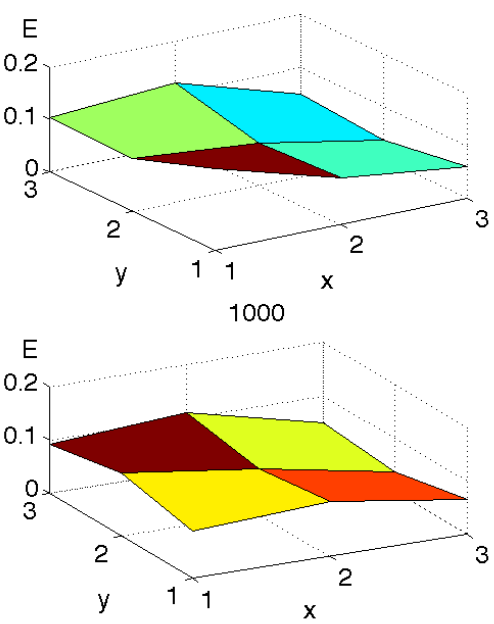

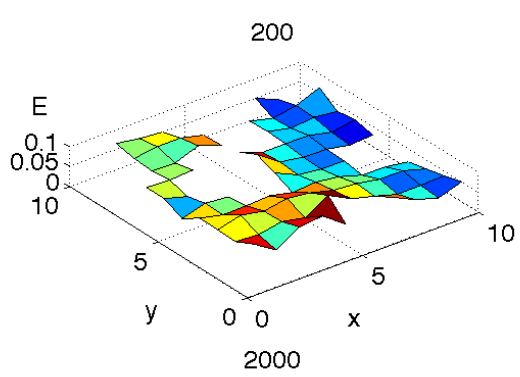

4: Network error, $10 \times 10,10 D$ feature vector

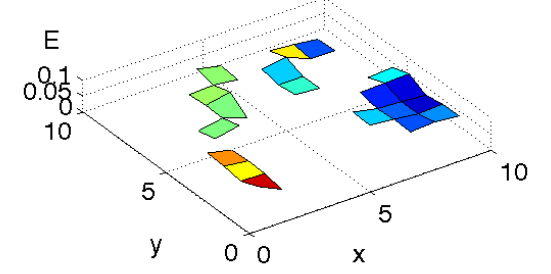




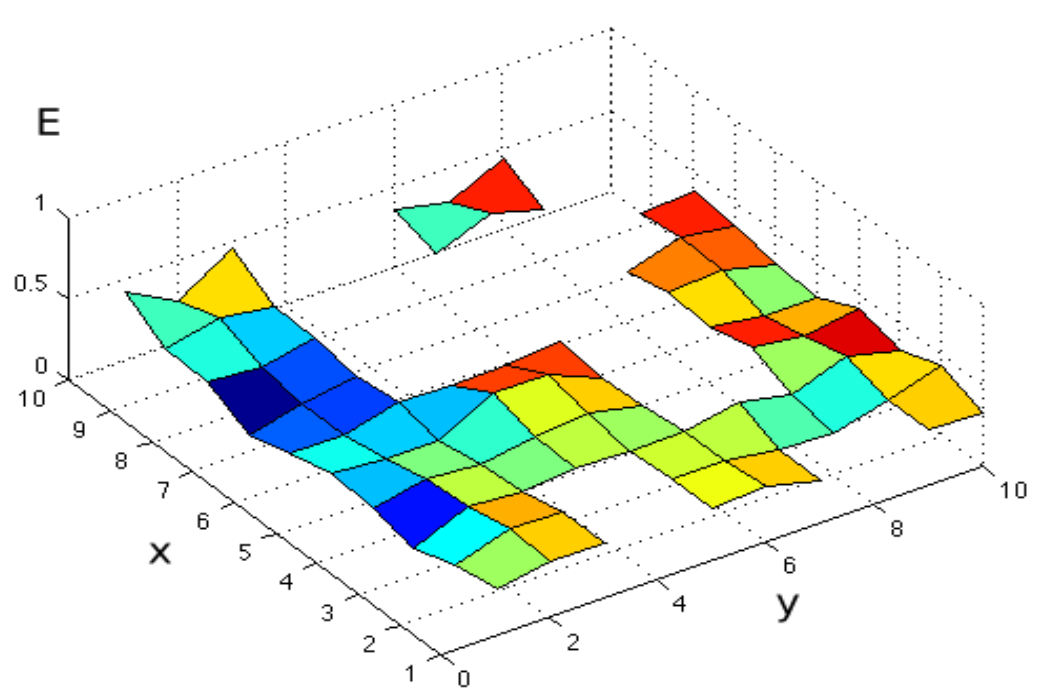

5: Network error, $10 \times 10,10 D$ feature vector, 3000 iterations, normalization
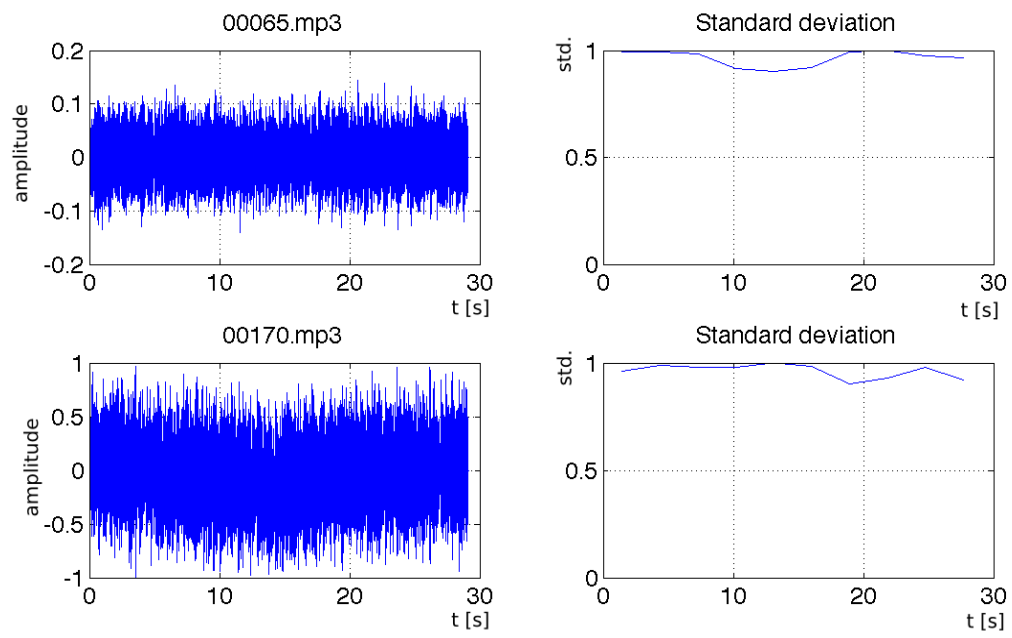

6: Observation 1 and 2 of neuron 61 (the smallest error)
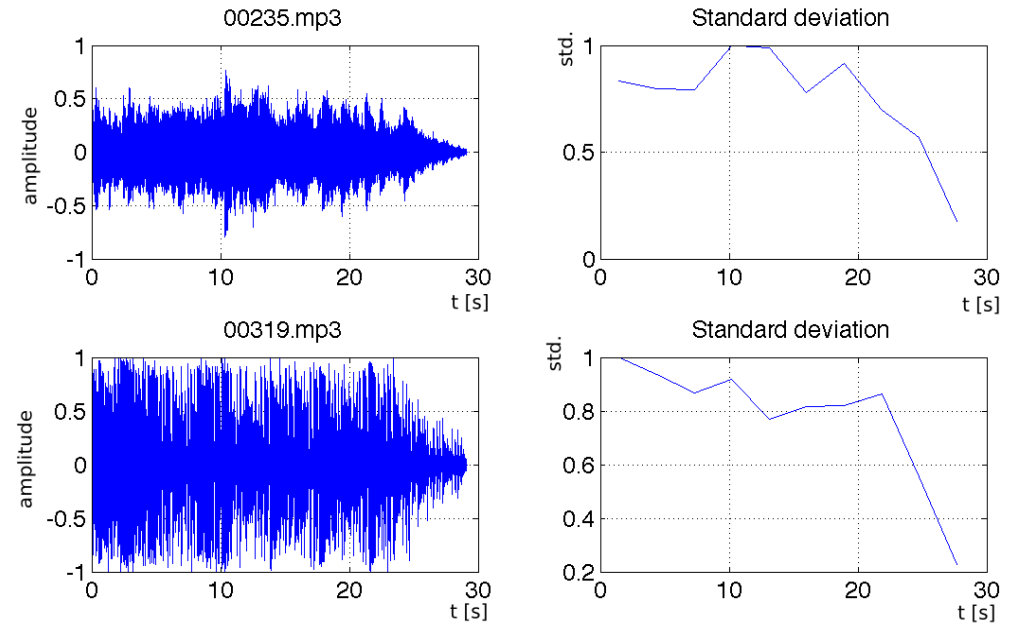

7: Observation 1 and 2 of neuron 96 ("fade out") 
most all observations. Error level decreases rapidly around the 52nd iteration and between 130th and 140th iteration. We thing, that these breaks represents local extremes overcoming. After that it remains at the same level.

Fig. 2. shows the same experiment with a 3 dimensional vector but with $10 \times 10$ topology. It can be seen that error level is approximately on the same level, but clustering is smoother. Another fact is that unused neurons appear there - the white fields. Those neurons classified nothing or only one observation (recording), so our error value determination has no meaning there.

Fig. 3. shows a 10 dimensional vector with $3 \times 3$ topology and finally Fig. 4. shows a 10 dimensional feature vector with 10 x 10 neurons topology.
For our purpose the most interesting option is 10 $\mathrm{x} 10$ neurons with the 10 dimensional feature vector with 2000 of learning iterations. There we can observe several natural clusters, which grew out from the data independently on number of neurons. This is different from the previous $3 \times 3$ topology (forced 9 classes) and also different from the popular clustering algorithm k-means where we have to set the number of classes.

We can examine the resulting map deeply. In Fig. 5. we can see a network error when clustering normalized feature vectors. Fig. 6. shows the first two observations classified by the best neuron number 61.

Fig. 7. shows the first two observations of neuron 96 which is situated in a detached group of neurons. A different type of time series appears here.

\section{SUMMARY}

This paper describes our experiment in the unsupervised clustering of a large time series database. It contains 1024 clips of audio recordings. Time series (feature vectors) are produced from these clips using a simplistic signal processing method. The influence of a time series dimensionality together with the number of neurons in the SOM is discussed. A network error based on the average Euclidian distance of observations from the mean of the cluster is evaluated and visualized for 4 configurations combining two types of the feature vector and two types of the SOM. It is shown that for clustering is the most interesting option a 10 dimensional feature vector clustered with a large SOM. There is performed normalization of feature vectors and results of clustering after 3000 learning iterations in batch mode are presented. We are presenting two examples classified by the best neuron with the smallest error and other two examples classified by neuron adherent to a detached group of neurons. As we can see in the Fig. 6 and 7 the development of the standard deviation descriptor is similar for each pair of recordings. And also recordings in detached clusters are different as we expected. This shows the usability of this algorithm for musical recordings clustering. Our research in time series clustering will continue in using supervised learning (LVQ) and semi-supervised learning in an effort to control the map layout.

This paper is supported by IGA project 64/2010.

\section{REFERENCES}

ABDALLAH, S. A., PLUMBLEY, M. D., 2007: Information Dynamics. Technical Report C4DM-TR07-01, Centre for Digital Music, Queen Mary University of London.

FEJFAR, J., WEINLICHOVÁ, J., ŠŤASTNÝ, J., 2010: Musical Form Retrieval. In: MENDEL 2010, 16th International Conference on Soft Computing. Brno: Brno University of Technology. ISSN 1803-3814.

KOHONEN, T., 2001: Self-Organizing Maps. Secaucus, NJ, USA: Springer-Verlag New York, Inc. ISBN 3540679219.

LAW, E., VON AHN, L., 2009: Input-agreement: A New Mechanism for Data Collection using Human
Computation Games. Proc. of CHI, Boston, Massachusetts, USA. ACM press 978-1-60558-247-4, pp. 1197-1206.

ŠTENCL, M., ŠŤASTNÝ, J., 2009: Advanced approach to numerical forecasting using artificial neural networks. Acta Universitatis agriculturae et silviculturae Mendelianae Brunensis, sv. 6, č. 2, pp. 297-304, ISSN 1211-8516.

WEI, L., KEOGH, E. J., 2006: Semi-supervised time series classification. In: Proceedings of the Twelfth ACM SIGKDD International Conference on Knowledge Discovery and Data Mining, Philadelphia, PA, USA. ISBN 1-59593-339-5.

\section{Address}

Ing. Jiří Fejfar, doc. RNDr. Ing. Jiří Štastný, CSc., Ústav informatiky, Mendelova Univerzita v Brně, Zemědělská 1, 61300 Brno, Česká republika, e-mail: jiri.stastny@mendelu.cz. 
